\title{
Optimal protein intake during pregnancy for reducing the risk of fetal growth restriction: the Japan Environment and Children's Study
}

\author{
Naho Morisaki ${ }^{1}$, Chie Nagata ${ }^{2}$, Shinobu Yasuo ${ }^{3 *}$, Seiichi Morokuma ${ }^{4}$, Kiyoko Kato ${ }^{5}$, Masafumi Sanefuji ${ }^{4}$, \\ Eiji Shibata $^{6}$, Mayumi Tsuji ${ }^{7}$, Ayako Senju ${ }^{6}$, Toshihiro Kawamoto ${ }^{7}$, Shouichi Ohga ${ }^{8}$, Koichi Kusuhara ${ }^{9}$ and \\ Japan Environment and Children's Study Group \\ ${ }^{1}$ Department of Social Medicine, National Center for Child Health and Development, 2-10-1, Okura, Setagaya-ku, \\ Tokyo 157-8535, Japan \\ ${ }^{2}$ Department of Education for Clinical Research, National Center for Child Health and Development, 2-10-1, Okura, \\ Setagaya-ku, Tokyo 157-8535, Japan \\ ${ }^{3}$ Laboratory of Regulation in Metabolism and Behavior, Faculty of Agriculture, Kyushu University, Hakozaki 6-10-1, \\ Higashi-ku, Fukuoka 812-8581, Japan \\ ${ }^{4}$ Research Center for Environmental and Developmental Medical Sciences, Kyusyu University, Fukuoka, Hakozaki 6-10-1, \\ Higashi-ku, Fukuoka 812-8581, Japan \\ ${ }^{5}$ Department of Obstetrics and Gynecology, Graduate School of Medical Sciences, Kyushu University, Hakozaki 6-10-1, \\ Higashi-ku, Fukuoka 812-8581, Japan \\ ${ }^{6}$ Regional Center for Japan Environment and Children's Study (JECS), University of Occupational and Environmental Health, \\ 1-1, Iseigaoka, Yahatanishi, Kitakyusyu 807-8556, Japan \\ ${ }^{7}$ Department of Environmental Health, School of Medicine, University of Occupational and Environmental Health, 1-1, \\ Iseigaoka, Yahatanishi, Kitakyusyu 807-8556, Japan \\ ${ }^{8}$ Department of Pediatrics, Graduate School of Medical Sciences, Kyusyu University, Hakozaki 6-10-1, Higashi-ku, \\ Fukuoka 812-8581, Japan \\ ${ }^{9}$ Department of Pediatrics, School of Medicine, University of Occupational and Environmental Health, 1-1, Iseigaoka, \\ Yahatanishi, Kitakyusyu 807-8556, Japan
}

(Submitted 8 March 2018 - Final revision received 20 August 2018 - Accepted 3 September 2018)

\section{Abstract}

Clinical trials show that protein supplement increases infant size in malnourished populations; however, epidemiological studies in highincome countries have reported mixed results. Although these findings suggest a non-linear relationship between maternal macronutrient intake and fetal growth, this relationship has not been closely examined. We assessed the association between maternal protein intake and fetal growth among 91637 Japanese women with singletons in a nation-wide cohort study using validated FFQ. The respondents answered the FFQ twice, once during early pregnancy (FFQ1; 16.3 (SD 6.0) weeks), and second during mid-pregnancy (FFQ2, 28.1 (SD 4.1) weeks). Daily energy intake and percentage energy from protein, fats and carbohydrates were 7477 (SD 2577) kJ and 13.5 (SD 2.0), 29.5 (sD 6.5) and 55.3 (sD 7.8) \%, respectively, for FFQ1, and 7184 (sD 2506) kJ and 13.6 (sD 2.1), $29 \cdot 8$ (sD 6.6) and $55 \cdot 3$ (sD 7.9) \%, respectively, for FFQ2. The average birth weight was 3028 (SD 406) g, and 6350 infants (6.9\%) were small for gestational age (SGA). In both phases of the survey, birth weight was highest and the risk of SGA was lowest when the percentage energy from protein was $12 \%$, regardless of whether isoenergetic replacement was with fat or carbohydrates. Furthermore, when protein density in the maternal diet was held constant, birth weight was highest when $25 \%$ of energy intake came from fat and $61 \%$ came from carbohydrates during early pregnancy. We found maternal protein intake to have an inverse U-curve relationship with fetal growth. Our results strongly suggest that the effect of protein on birth weight is non-linear, and that a balanced diet fulfilling the minimum requirement for all macronutrients was ideal for avoiding fetal growth restriction.

Key words: Protein: Macronutrients: Maternal diet: Fetal growth: Small-for-gestational age

\footnotetext{
Abbreviations: JECS, Japan Environment and Children's Study; SGA, small for gestational age.
}

* Corresponding author: S. Yasuo, fax +8192642 4426, email syasuo@brs.kyushu-u.ac.jp 
Adequate nutrition during pregnancy is crucial for fetal development. The theory of the fetal origins of adult disease has renewed interest in nutrition during pregnancy. A study of survivors of the Dutch famine demonstrated that low birth weight due to maternal malnutrition increased infant susceptibility to CVD and type 2 diabetes in adulthood ${ }^{(1,2)}$. Among the nutrient groups assessed, proteins have emerged as an important factor related to birth weight.

Studies on protein intake during pregnancy suggested that while fulfilling the minimum protein requirement may help prevent fetal growth restriction, a higher protein intake may be more harmful than beneficial. A rodent model has repeatedly demonstrated that protein deprivation can hamper fetal growth $^{(3-6)}$ and cause permanent changes in the structure of tissues and organs and physiological functions in offspring $^{(1,3,6-8)}$. In low- and middle-income countries, as well as in populations where malnutrition is common, protein supplementation has been shown to reduce the risk of low birth weight and small for gestational age (SGA) births ${ }^{(9-11)}$. However, randomised interventional studies using supplementation with excessive protein ( $>20 \%$ of energy as protein) have repeatedly shown that increased protein intake decreased fetal growth $^{(12,13)}$, especially in high-income countries.

Based on evidence that both high and low protein intake may reduce fetal growth, current recommendations suggest a maternal dietary protein intake of $10-20 \%$ of the total energy intake $^{(14)}$. However, the 'optimal' protein intake for pregnant women is unknown. The interventional studies on this topic have thus far been two-armed and therefore unsuitable for detailed calculation. Most previous epidemiological studies have proposed nothing more complex than a linear association between protein intake and birth outcomes with inconsistent results. We were able to find only two studies, one from New Zealand with 504 women $^{(15)}$ and another from Australia with 1040 women $^{(16)}$, which suggested a non-linear relationship between maternal macronutrient intake and fetal growth. However, while both studies found that a quadratic model fit better than a linear model, the studies were relatively small and had insufficient power to produce conclusive results. Furthermore, most research on maternal macronutrients has focused on protein, and only a few studies have examined the importance of the other two macronutrients, that is, carbohydrates and fats.

Using data from a nationwide cohort study involving nearly 100000 women and the cubic-spline model, we evaluated the association between maternal macronutrient intake and fetal growth.

\section{Methods \\ Study sample}

This study was based on data obtained from the Japan Environment and Children's Study (JECS), a Japan-wide prospective cohort study of pregnant women, their spouses and their children ${ }^{(17,18)}$. Pregnant women in any of the fifteen study regions throughout Japan were recruited from January 2011 to March 2014 either at their first antenatal visit at the participating, local health care institution or at a local government office issuing the socalled Mother and Child Health Handbook, a booklet issued to all expecting mothers in Japan enabling them to receive municipal health care services for their pregnancy, delivery and childcare. Women who were unable to participate in the survey or had difficulty filling out the questionnaire in Japanese were excluded. During pregnancy, the participants were asked to respond to two surveys covering demographics, life style, behaviour and medical history; one questionnaire was administered at recruitment (MT1) and the other was administered later during the pregnancy (MT2). Both surveys included a FFQ. Birth characteristics and medical information were collected separately from the medical records (DrOm).

For this study, we used the dataset for birth outcomes 'jecs-ag-20160424', which was created in April 2016 and revised in October 2016. From the 104102 births, we excluded multiple pregnancies, preterm deliveries before 28 weeks, post-term deliveries after 42 weeks, births with missing background characteristics and births from heavily obese women (BMI $>35 \mathrm{~kg} / \mathrm{m}^{2}$ ). In total 91637 (90\%) subjects were enrolled (Fig. 1). Among these, 79578 (87\%) underwent gestational weight gain measurement at 6-14 weeks and had answered the first FFQ (daily total energy intake of 2092-18828 kJ); 82250 (89\%) underwent a gestational weight gain measurement at 20-28 weeks and had answered the second FFQ (daily total energy intake of 2092-18828 kJ).

\section{FFQ}

Our FFQ, listing 172 food and beverage item, asked about respondents' habitual consumption of the listed food items using three portion sizes and nine frequency categories ${ }^{(19)}$. The FFQ has been previously validated using 3-d dietary records and blood samples ${ }^{(20,21)}$ in adults, but not specifically in pregnant women. The intake of energy was calculated by food group using a food composition table developed for the FFQ based on the Standardized Tables of Food Composition developed in Japan (2010 edition). The first FFQ (FFQ1) was administered during early pregnancy and asked about respondents' diet over the past year while the second FFQ (FFQ2) was administered during mid pregnancy and asked about respondents' diet during pregnancy (from conception to the answering date).

Protein, fat and carbohydrate density was calculated as a percentage of the energy intake from that nutrient; for example, protein density was calculated using the formula: (protein intake $(\mathrm{g})$ reported in $\mathrm{FFQ} \times 4(\mathrm{~kJ} / \mathrm{g})$ /energy intake $(\mathrm{kJ})$ reported in FFQ).

\section{Demographic data}

Maternal socio-demographic data collected from the responses to the second questionnaire were categorised by annual household income ( $<2$ million yen, 2-4 million yen, 4-6 million yen, 6-8 million yen, $>8$ million yen and no answer); maternal education (university education or higher, 2-year college, vocational school, high school or lower) and smoking status 


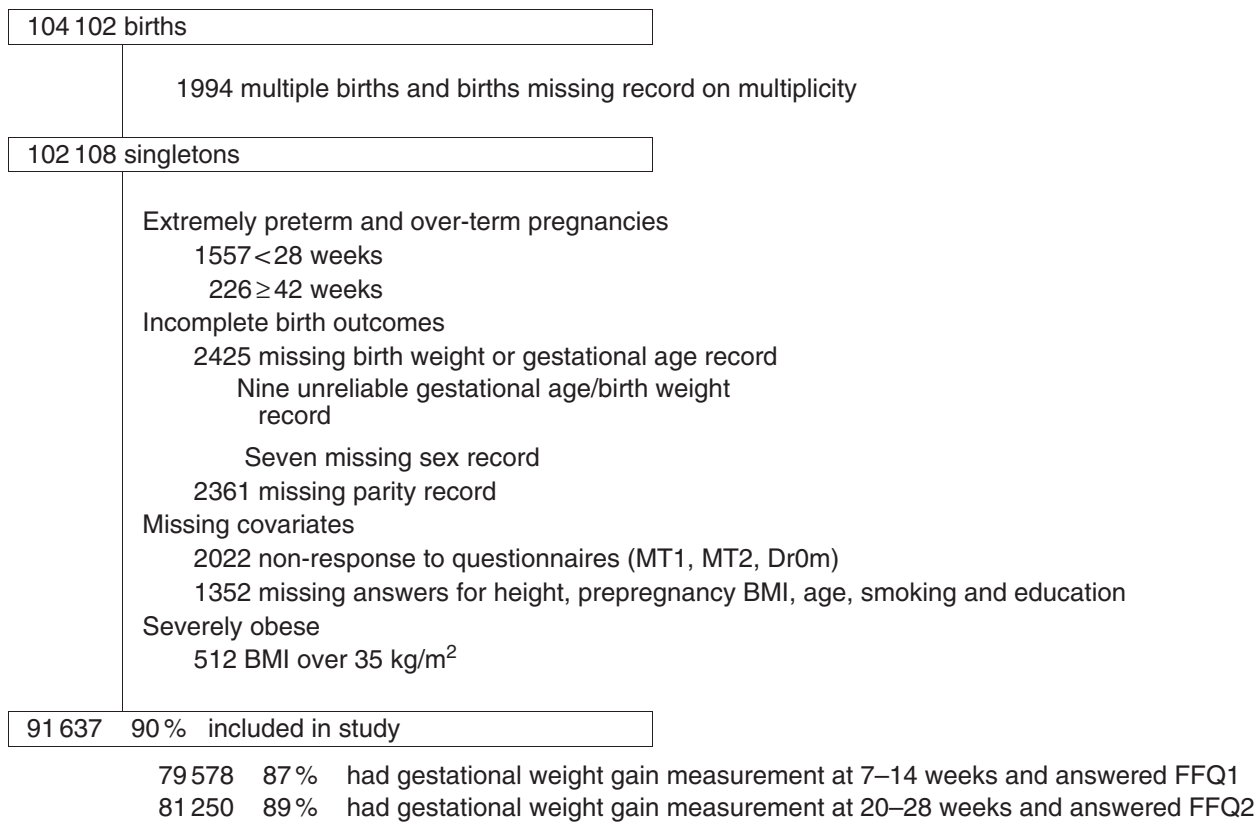

Fig. 1. Population flow chart.

(never smoked, previously smoked but stopped before pregnancy, previously smoked but stopped because of pregnancy and current smoker). Pre-pregnancy BMI was calculated from height and pre-pregnancy weight (either self-reported or measured) and categorised as under $18.5,18.5-25$ or $25 \mathrm{~kg} / \mathrm{m}^{2}$ or higher. Weight measurements at the antenatal check-up visit at 7-14 and 20-28 weeks were collected from the Mother-Child Health Handbook and used to calculate gestational weight gain during early and mid-pregnancy.

Other baseline characteristics retrieved from the medical records were maternal age $\left(<25,25-34, \geq 35 \mathrm{~kg} / \mathrm{m}^{2}\right)$ and parity ( 0,1 or more). Data on birth outcomes (gestational age and birth weight) were also obtained from the medical records. SGA was defined as a birth weight below the tenth percentile of the normal population at each day of gestation and was stratified by sex and parity using the Japanese birth weight reference chart $^{(22)}$. The same chart was used to calculate the birth weight $z$-score for each infant.

\section{Ethics}

The JECS protocol was approved by the review board for epidemiological studies of the Ministry of the Environment and by the Ethics Committees of all the participating institutions. The JECS protocol was conducted in accordance with the Helsinki Declaration and other nationally valid regulations, and written informed consent was obtained from each participant.

\section{Statistics}

We calculated the average birth weight and proportion of SGA of infants based on the characteristics of the study population and the average dietary profile of the respondents to the two FFQ.
Next, we used the cubic spline model to analyse the association between maternal macronutrient intake and birth outcomes, namely, birth weight and risk of SGA. In the restricted cubic spline, all data points are used to estimate the doseresponse association between the continuous independent variable and the outcome, thus allowing non-linear associations. The OR (for SGA) and change in birth weight, as well as their accompanying 95\% CI, were calculated with four knots placed at the 5th, 50th, 85th and 95th percentiles of the density of each macronutrient.

As our primary exposure of interest was maternal protein intake, our main analysis focused on the association of protein density in the maternal diet as assessed by FFQ1 (of the prepregnancy period to the first trimester) with birth weight and the risk of SGA. To evaluate the effect of protein relative to other macronutrients in an isoenergetic diet, a substitution model using the multivariate nutrient density method was employed ${ }^{(23)}$.

Next, we created a model in which both energy and protein density were constant in order to observe the association between carbohydrate density and fetal growth when isoenergetic replacement was with fat, and conversely the association between fat density and fetal growth when isoenergetic replacement was with carbohydrates.

Due to the possibility that some women may have modified their diet after becoming pregnant, we conducted a sensitivity analysis using the maternal diet as assessed in FFQ2 (covering diet from conception to the second trimester).

Maternal age, parity, education, income, prepregnancy BMI, height, smoking status and infant sex were treated as potential confounders in all our analyses, and adjustments were made for recruitment site, total energy intake as reported in the FFQ, and gestational weight gain and age at the time of the survey (which also reflected energy intake and contained fewer measurement errors than the total energy value reported in the FFQ). 
All statistical analyses were conducted using the statistical software package Stata 13 (Stata Corp). A $P$-value $<0.05$ was considered to be statistically significant when performing hypothesis tests. The spline curve was constructed using the 'mkspline' command in STATA. As this was a secondary analysis of a large cohort study, sample size calculation was not conducted before analysis.

\section{Results}

Table 1 shows the birth outcomes according to the characteristics of the study population. The average birth weight was 3028 (sD 406) g, and 6350 (6.9\%) births were SGA. The proportion of SGA births was highest among women who were shorter, thinner or continued to smoke during pregnancy. The proportion of SGA births was also higher among women from a lower income or educational level, older women, primiparae and women with low gestational weight gain until 20-28 weeks.

Table 2 shows the nutritional characteristics. The mean daily energy intake and percentage energy from protein, fats and carbohydrates were 1787 (7477 (sD 2577) kJ and 13.5 (sD 2.0), 29.5 (SD 6.5) and 55.3 (SD 7.8) \%, respectively, for FFQ1, and 7184 (sD 2506) kJ and 13.6 (sD 2.1), 29.8 (sD 6.6) and 55.3 (sD 7.9) $\%$, respectively, for FFQ2. The mean gestational weight gain for measurements when the two questionnaire were answered were $0.5(\mathrm{sD} 2 \cdot 0)$ and $11 \cdot 1$ (sD 1.6) kg, respectively.

The cubic-spline model demonstrated a dose-response relationship between maternal protein density as reported in FFQ2 and birth outcomes after adjusting for maternal

Table 1. Birth weight and fetal growth by characteristic ( $n 91637$ )

(Numbers and percentages; mean values and standard deviations)

\begin{tabular}{|c|c|c|c|c|c|c|}
\hline & \multirow[b]{2}{*}{$n$} & \multirow[b]{2}{*}{$\%$} & \multicolumn{2}{|c|}{ Birth weight $(\mathrm{g})$} & \multicolumn{2}{|c|}{ SGA } \\
\hline & & & Mean & SD & $n$ & $\%$ \\
\hline \multicolumn{7}{|l|}{ Maternal age (years) } \\
\hline$<25$ & 10382 & $11 \cdot 3$ & 3026 & 389 & 703 & $6 \cdot 8$ \\
\hline $25-34$ & 59120 & 64.5 & 3033 & 399 & 4076 & 6.9 \\
\hline$\geq 35$ & 22135 & $24 \cdot 2$ & 3019 & 429 & 1571 & $7 \cdot 1$ \\
\hline \multicolumn{7}{|l|}{ Birth order } \\
\hline First child & 36891 & $40 \cdot 3$ & 2996 & 406 & 2669 & $7 \cdot 2$ \\
\hline Not first child & 54746 & $59 \cdot 7$ & 3050 & 404 & 3681 & 6.7 \\
\hline \multicolumn{7}{|l|}{ Height $(\mathrm{cm})$} \\
\hline $130-155$ & 28951 & 31.6 & 2956 & 399 & 2661 & 9.2 \\
\hline $156-160$ & 33618 & $36 \cdot 7$ & 3030 & 398 & 2206 & $6 \cdot 6$ \\
\hline $161-183$ & 29068 & $31 \cdot 7$ & 3098 & 408 & 1483 & $5 \cdot 1$ \\
\hline \multicolumn{7}{|l|}{ Prepregnancy BMI $\left(\mathrm{kg} / \mathrm{m}^{2}\right)$} \\
\hline$<18.5$ & 14839 & $16 \cdot 2$ & 2927 & 387 & 1526 & $10 \cdot 3$ \\
\hline $18 \cdot 5-24.9$ & 67452 & $73 \cdot 6$ & 3038 & 397 & 4355 & 6.5 \\
\hline$\geq 25$ & 9346 & $10 \cdot 2$ & 3122 & 459 & 469 & $5 \cdot 0$ \\
\hline \multicolumn{7}{|c|}{ Annual household income (millions of yen) } \\
\hline$<2$ & 4798 & $5 \cdot 2$ & 3014 & 418 & 376 & 7.8 \\
\hline 2 to $<4$ & 29484 & $32 \cdot 2$ & 3027 & 409 & 2088 & $7 \cdot 1$ \\
\hline 4 to $<6$ & 28349 & $30 \cdot 9$ & 3031 & 401 & 1872 & 6.6 \\
\hline 6 to $<8$ & 13677 & 14.9 & 3031 & 404 & 938 & 6.9 \\
\hline$\geq 8$ & 9273 & $10 \cdot 1$ & 3032 & 406 & 627 & $6 \cdot 8$ \\
\hline No response & 6056 & $6 \cdot 6$ & 3026 & 406 & 449 & 7.4 \\
\hline \multicolumn{7}{|l|}{ Highest maternal education } \\
\hline High school or less & 33152 & $36 \cdot 2$ & 3027 & 411 & 2389 & $7 \cdot 2$ \\
\hline Vocational school & 22469 & 24.5 & 3026 & 406 & 1599 & $7 \cdot 1$ \\
\hline 2-year college & 16172 & $17 \cdot 6$ & 3029 & 405 & 1075 & 6.6 \\
\hline University or higher & 19844 & $21 \cdot 7$ & 3033 & 397 & 1287 & 6.5 \\
\hline \multicolumn{7}{|l|}{ Smoking status } \\
\hline Never smoked & 53003 & $57 \cdot 8$ & 3027 & 403 & 3646 & 6.9 \\
\hline Stopped before pregnancy & 21991 & $24 \cdot 0$ & 3048 & 405 & 1361 & $6 \cdot 2$ \\
\hline Stopped because of pregnancy & 12443 & $13 \cdot 6$ & 3037 & 412 & 823 & $6 \cdot 6$ \\
\hline Current smoker & 4200 & 4.6 & 2912 & 407 & 520 & $12 \cdot 4$ \\
\hline \multicolumn{7}{|c|}{ Gestational weight gain measured at $7-14$ weeks $(\mathrm{kg})^{\star}$} \\
\hline$<0$ & 26776 & $29 \cdot 2$ & 3021 & 397 & 1835 & 6.9 \\
\hline 0 to $<2$ & 37315 & $40 \cdot 7$ & 3027 & 403 & 2651 & 7.1 \\
\hline$\geq 2$ & 16657 & $18 \cdot 2$ & 3059 & 413 & 1020 & $6 \cdot 1$ \\
\hline \multicolumn{7}{|c|}{ Gestational weight gain measured at $20-28$ weeks $(\mathrm{kg}) \dagger$} \\
\hline$<4$ & 25901 & $28 \cdot 3$ & 2976 & 409 & 2311 & 8.9 \\
\hline 4 to $<7$ & 34828 & $38 \cdot 0$ & 3027 & 395 & 2361 & $6 \cdot 8$ \\
\hline$\geq 7$ & 21675 & $23 \cdot 7$ & 3103 & 403 & 1002 & 4.6 \\
\hline \multicolumn{7}{|l|}{ Infant sex } \\
\hline Male & 46942 & $51 \cdot 2$ & 3071 & 411 & 3242 & 6.9 \\
\hline Female & 44695 & $48 \cdot 8$ & 2983 & 395 & 3108 & $7 \cdot 0$ \\
\hline
\end{tabular}

SGA, small for gestational age.

* Among 80748 with weight measurement at 7-14 weeks.

† Among 82404 with weight measurement at 20-28 weeks. 
Table 2. Dietary profile of pregnant women during pregnancy (Mean values and standard deviations)

\begin{tabular}{|c|c|c|c|c|}
\hline & \multicolumn{2}{|c|}{$\mathrm{FFQ1}^{\star}(n$ 80748) } & \multicolumn{2}{|c|}{ FFQ2† ( $n$ 82 404) } \\
\hline & Mean & SD & Mean & SD \\
\hline Gestational age at survey (weeks) & $16 \cdot 3$ & 6.0 & $28 \cdot 1$ & 4.1 \\
\hline Daily energy intake $(\mathrm{kJ})$ & $7475 \cdot 1$ & $2575 \cdot 7$ & $7183 \cdot 1$ & $2505 \cdot 0$ \\
\hline Daily protein intake $(\mathrm{g})$ & $61 \cdot 2$ & $25 \cdot 6$ & 58.9 & $25 \cdot 1$ \\
\hline Energy from protein (\%) & 13.5 & 2.0 & $13 \cdot 6$ & $2 \cdot 1$ \\
\hline Daily fat intake $(\mathrm{g})$ & 59.9 & 28.4 & $58 \cdot 2$ & $27 \cdot 9$ \\
\hline Energy from fat (\%) & 29.5 & 6.5 & $29 \cdot 8$ & $6 \cdot 6$ \\
\hline Daily carbohydrates intake $(\mathrm{g})$ & 243.4 & $80 \cdot 2$ & 233.7 & $77 \cdot 0$ \\
\hline Energy from carbohydrates (\%) & $55 \cdot 3$ & 7.8 & $55 \cdot 3$ & 7.9 \\
\hline
\end{tabular}

* FFQ1 was administered during early pregnancy and asked about respondents' diet over the previous year. † FFQ2 was administered during mid pregnancy and asked about the respondents' diet during pregnancy.
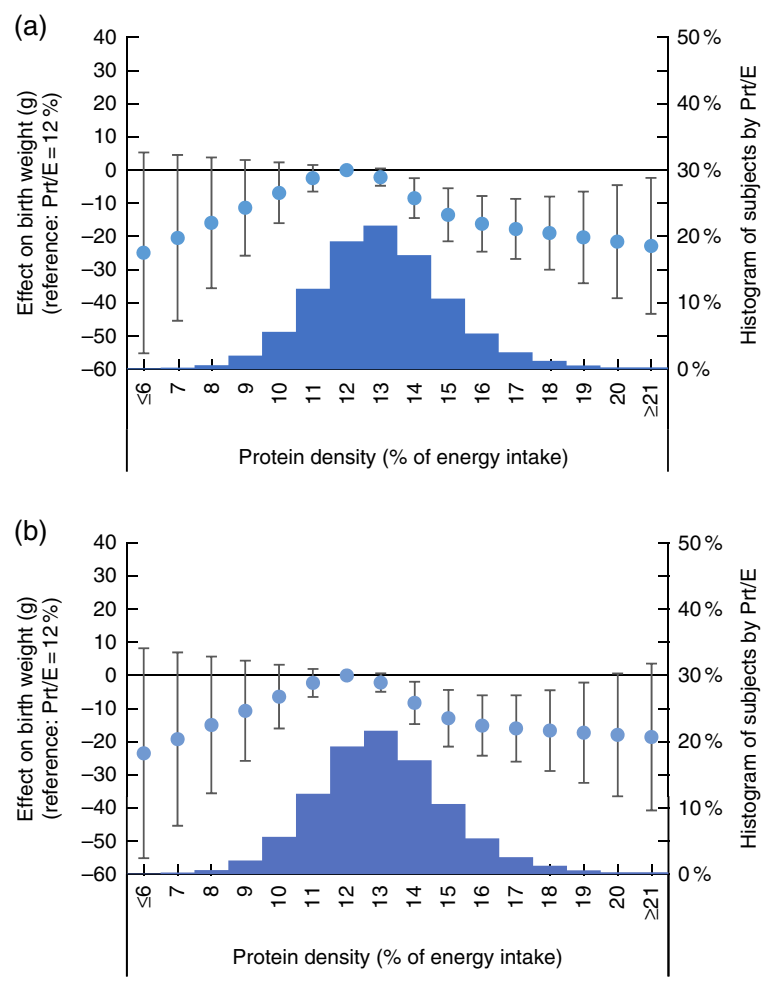

Fig. 2. Association between dietary protein density as assessed in FFQ1 and birth weight. (a) Analysis with isoenergetic replacement of protein with carbohydrate. (b) Analysis with isoenergetic replacement of protein with fat. Prt/E, protein energy percentage. Histogram displays number of subjects by dietary protein density as assessed in FFQ1. Estimated difference in birth weight compared with women who had highest birth weight is shown in circle, with associated $95 \% \mathrm{Cl}$ shown as whiskers.

characteristics and energy intake. The accompanying histograms showing the distribution of protein density are shown in Fig. 2 (for birth weight) and Fig. 3 (for risk of SGA). The reference level of the spline models was set at a protein density of $12 \%$. Fig. 2 shows that birth weight was highest when percentage energy from protein was $12 \%$, regardless of whether isoenergetic replacement was with fat or carbohydrates. Women whose diet contained a protein density above $14 \%$ showed a significantly lower birth weight than those whose protein density was $12 \%$. Similarly, Fig. 3 shows a U-curve relationship between protein density and the risk of SGA; the
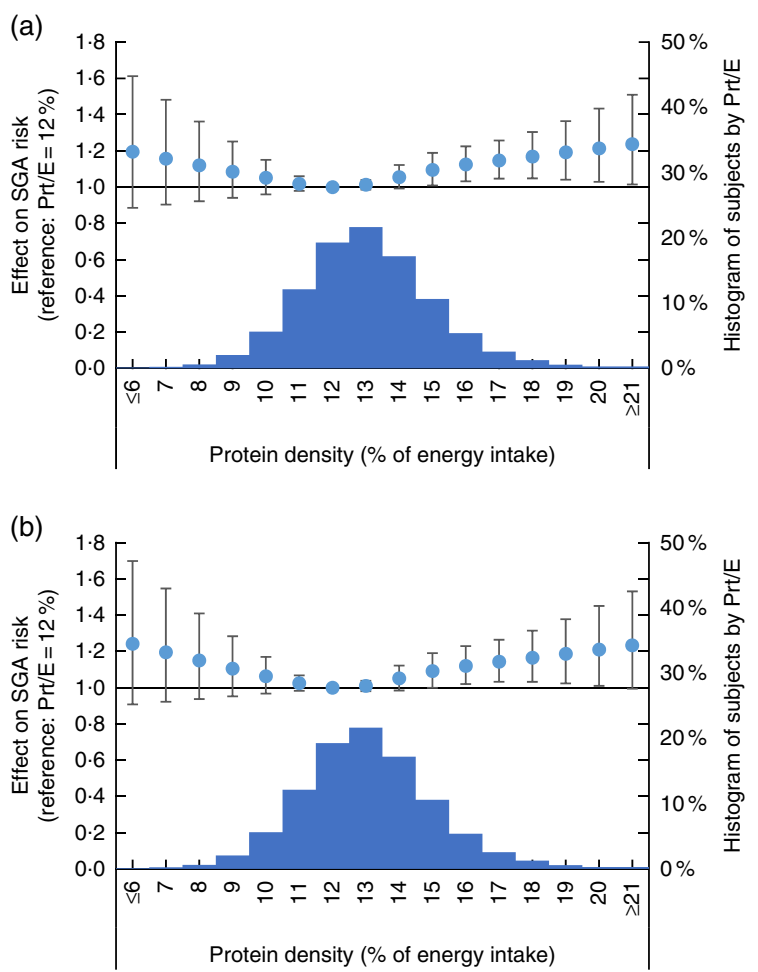

Fig. 3. Association between dietary protein density as assessed in FFQ1 and risk of small for gestational age (SGA) birth. (a) Analysis with isoenergetic replacement of protein with carbohydrate. (b) Analysis with isoenergetic replacement of protein with fat. Prt/E, protein energy percentage. Histogram displays number of subjects by dietary protein density as assessed in FFQ1. Estimated OR of SGA compared with women who had lowest risk of SGA is shown in circle, with associated $95 \% \mathrm{Cl}$ shown as whiskers.

latter was lowest when percentage energy from protein was $12 \%$, regardless of whether isoenergetic replacement was with fat or carbohydrates. Women whose diet contained a protein density above $15 \%$ showed a significantly higher risk of SGA compared with those whose protein density was $12 \%$. The shape of the association between dietary protein density and birth outcomes was similar to that of the maternal diet as assessed in FFQ1 (online Supplementary Appendix S1).

Fig. 4(a) shows the association between dietary carbohydrate density with birth weight while Fig. 4(b) shows the association between dietary fat density with birth weight. The 
(a)
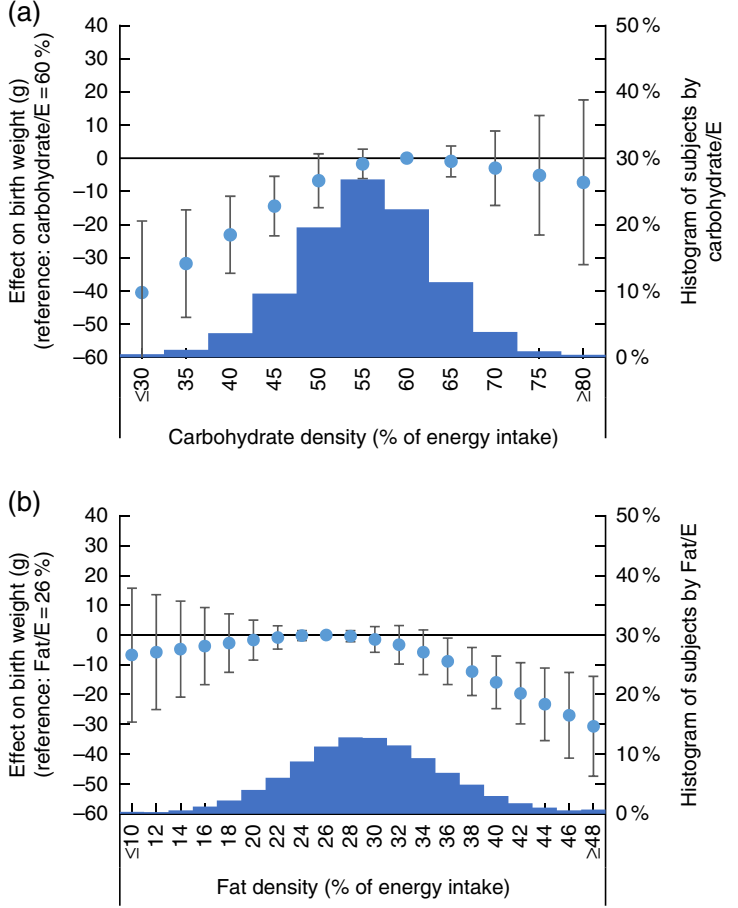

Fig. 4. Association between dietary fat and carbohydrate density as assessed in FFQ1 and birth weight. (a) Analysis with isoenergetic replacement of fat with carbohydrate. (b) Analysis with isoenergetic replacement of carbohydrate with fat. Fat $/ E$, fat energy percentage; carbohydrate/E, carbohydrate energy percentage. Histogram displays number of subjects by dietary protein density as assessed in FFQ1. Estimated difference in birth weight compared with women who had highest birth weight is shown in circle, with associated $95 \% \mathrm{Cl}$ shown as whiskers.

accompanying histograms show the macronutrient density distribution for each nutrient. All models were adjusted for maternal characteristics, energy intake and protein intake. Fig. 4(a) shows that women whose diet had fat density of $25 \%$ had highest birth weight, with women whose diet had fat density over $35 \%$ having significantly lower birth weight. Fig. 4(b) shows that women whose diet contained a carbohydrate density of $59 \%$ had highest birth weight, with women whose diet had carbohydrate density below $47 \%$ having significantly lower birth weight. On the other hand, no significant difference in birth weight by carbohydrate or fat density of the diet was observed for FFQ2 (online Supplementary Appendix S2).

For both questionnaires, $12 \%$ remained the protein density with highest birth weight in crude analysis not adjusting for possible confounders, and after excluding 2949 women who were diagnosed who developed gestational diabetes during pregnancy (results not shown).

\section{Discussion}

Among Japanese women, the association between protein intake and risk of fetal growth restriction described a U-shaped curve, suggesting that both high and low protein intake can increase its risk. Our study demonstrated that fetal growth peaked when protein density in the maternal diet was $12 \%$. This association was consistent for dietary reporting both in early pregnancy (covering diet over the past year) and in midpregnancy (covering diet during pregnancy). We also found that when protein intake was constant, birth weight decreased if $<29 \%$ of the energy intake derived from fat or more than $59 \%$ derived from carbohydrates.

Most previous epidemiological studies have failed to consider anything more complex than a linear association between protein intake and birth outcomes. Their findings were also inconclusive. Studies conducted in the $\mathrm{UK}^{(8)}$, Australia ${ }^{(16,24)}$ and Spain ${ }^{(25)}$ reported that higher protein density in the maternal diet was associated with a linear increase in birth weight; however, studies in the USA demonstrated that a higher protein intake was associated with reduced fetal growth ${ }^{(12,13,26)}$. Other studies ${ }^{(27-30)}$ failed to find any significant linear association. The present study suggests that the relationship is not linear but U-shaped; hence the discrepancies with previous studies may have resulted in part from differences in the mean protein intake in the population (e.g., women in the USA generally have a high protein intake ${ }^{(26)}$. Such interpretations have been proposed in smaller studies ${ }^{(12,15)}$ but have not been confirmed.

We believe our study is the first to calculate the optimal protein density for the maternal diet during pregnancy. The cubic-spline model and a sufficient sample size allowed us to express the association between protein intake and fetal growth in a nonlinear fashion. We found that while an increase in protein density up to $12 \%$ was associated with increased fetal growth and reduced SGA, any further increase in protein density significantly reduced fetal growth. Our findings did not conflict with those of interventional studies showing that while balanced protein supplementation (10-20\% of energy as protein) promoted fetal growth, especially in malnourished populations $^{(9-11,31)}$, supplementation with excessive protein ( $>20 \%$ of energy as protein) decreased fetal growth, especially in populations with a diet already high in protein density $^{(12,13)}$. Our results further suggested that the protein requirement was quite low, as we found that women who reported a diet containing a protein density of $14 \%$ in early pregnancy had a significantly lower birth weight and birth weight $z$-score than those who reported a protein density of $12 \%$.

Few studies have examined the influence of maternal carbohydrate and fat intake, compared with that of protein, on fetal growth. A cohort study in the UK found that low carbohydrate intake in early pregnancy was associated with lower methylation levels in DNA sequences regulating an imprinted gene associated with fetal growth but failed to find any association with birth weight ${ }^{(32)}$. Animal studies showed that a high maternal fat intake may increase adult body weight ${ }^{(33)}$; however, studies of birth weight have produced inconsistent findings ${ }^{(33)}$ as seen, for instance, in the conflicting reports of increased birth weight in mice ${ }^{(34)}$ and reduced birth weight in rats ${ }^{(35,36)}$. Our study demonstrated that lower fat and higher carbohydrate density in early-pregnancy significantly decreased risk of fetal growth. However, as these findings lack the support of previous epidemiological or experimental studies, the general validity of these findings needs to be weighed against the results of future studies. 


\section{Strengths and limitations}

The chief strength of our study is its large subject pool, which enabled us to examine the non-linear relationship between nutritional intake and birth outcomes. In addition, assessment of nutritional status was conducted twice during pregnancy, with the replication of the results providing greater reassurance of their reliability. However, there are several limitations. First, dietary assessments conducted during early pregnancy covered the previous year, which included the prepregnancy period in addition to early pregnancy. However, previous studies on the replication of nutritional assessment have shown that while absolute intake may change from prepregnancy to pregnancy (due to morning sickness) ${ }^{(37)}$ and the intake of certain micronutrients (such as folate) may change due to antenatal education, overall dietary preferences do not change ${ }^{(38)}$. Second, the reported total energy intake later in pregnancy (mean: $7184 \mathrm{~kJ} / \mathrm{d}$ ) was lower than in early pregnancy $(7477$ $\mathrm{kJ} / \mathrm{d}$ ), suggesting the possibility that women under-reported their energetic intake later in pregnancy possibly out of concern for social norms ${ }^{(39)}$ or reduced their intake in compliance with the strict national gestational guidelines ${ }^{(40)}$, which discourage excessive energetic intake during pregnancy. However, the adjustment for total energy intake in our analyses, the absence of any discernible reason for under-reporting macronutrient consumption disproportionately, the consistency of the association between macronutrient intake and fetal growth in the two FFQ, all suggest that this problem may not have had a significant influence on our results. Third, the association between macronutrients and the outcomes may have been affected by the intake of micro-nutrients correlating with the intake of macronutrients. However, the evidence from previous epidemiological and interventional studies using protein was consistent with our results. Similarly, while we cannot deny the fact there may be residual confounding due to unmeasured confounders, the stableness of the estimates which did not change much from the crude analysis to the adjusted analysis, as well as after excluding women with gestational diabetes suggest our results are likely to be robust. We thus believe that our interpretation of the inverse U-curve relationship of maternal dietary protein density with fetal growth is valid. Fourth, our sample population was limited to Japanese women, who generally have a different dietary culture than their Western counterparts and also eat less on the whole. Recent statistics (2010-2011) from the Japan National Health and Nutrition Survey based on 3-d dietary records ${ }^{(41)}$ show that consumption of both protein and total energy among pregnant women is very low ${ }^{(42)}$, with an average pregnant woman eating only 6966 (sD 1845) $\mathrm{kJ} / \mathrm{d}$ with a protein density of 13.8 (sD 4.3 ) \%, comparable to what we found in our population (7184 (SD 2506) kJ/d and 13.6 (sD 2.1)\%, respectively). Therefore, while our findings on the association between macronutrients and fetal growth is likely generalisable to the Japanese population or other populations with generally low energy intake, they may not be directly applicable to those who have a higher energy. Ideally, studies similar to the present one would be conducted in populations with different dietary cultures. Finally, while our study was able to calculate the 'optimal' ratio of the three nutrients to reduce risk of fetal growth restriction, it is much too early to interpret this as the 'optimal' maternal diet. The observed effect of protein intake on birth weight in this study is 10-20 g and quite small, thus if a different and more important outcome shows a different pattern with protein intake, such findings also need to be incorporated when deciding on what the true nutritional requirement is. Our study focused only on birth outcomes as the long-term outcomes of the cohort participants are not yet available, and mainly on fetal growth restriction as other birth outcomes such as neonatal deaths $(<0.1 \%)$, stillbirths $(0.3 \%)$ or macrosomia ( $0.6 \%$ had birth weight over $4 \mathrm{~kg}$ ) were extremely rare in the Japanese population. Future rigorous research using adequate databases should be conducted to estimate the influence of maternal nutrition on multiple outcomes, to provide the full picture of nutritional recommendations during pregnancy.

\section{Conclusion}

In conclusion, we found that protein intake from conception to the second trimester showed an inverse U-curve relationship with fetal growth. Our results strongly suggest that the association between protein intake and birth weight is nonlinear, and that a balanced diet fulfilling the minimum requirement for all macronutrients is ideal for avoiding fetal growth restriction.

\section{Acknowledgements}

The authors would like to express their gratitude to all the participants in this study and all the individuals involved in data collection. The authors would also like to thank the following members of the JECS (principal investigator, T. K.) as of 2017: Hirohisa Saito (National Center for Child Health and Development, Tokyo, Japan), Reiko Kishi (Hokkaido University, Sapporo, Japan), Nobuo Yaegashi (Tohoku University, Sendai, Japan), Koichi Hashimoto (Fukushima Medical University, Fukushima, Japan), Chisato Mori (Chiba University, Chiba, Japan), Shuichi Ito (Yokohama City University, Yokohama, Japan), Zentaro Yamagata (University of Yamanashi, Chuo, Japan), Hidekuni Inadera (University of Toyama, Toyama, Japan), Michihiro Kamijima (Nagoya City University, Nagoya, Japan), Takeo Nakayama (Kyoto University, Kyoto, Japan), Hiroyasu Iso (Osaka University, Suita, Japan), Masayuki Shima (Hyogo College of Medicine, Nishinomiya, Japan), Yasuaki Hirooka (Tottori University, Yonago, Japan), Narufumi Suganuma (Kochi University, Nankoku, Japan), K. K. (University of Occupational and Environmental Health, Kitakyushu, Japan) and Takahiko Katoh (Kumamoto University, Kumamoto, Japan).

JECS was funded by the Japanese Ministry of the Environment. The findings and conclusions of this article are solely the responsibility of the authors and do not represent the official views of the above government. This article was supported in part by the Grant-in-Aid for Young Scientists from the Japan Society for the Promotion of Science (26870889) to N. M. The funding bodies had no role in the design of the study, collection and analysis of data, interpretation of the results, writing of the manuscript or the decision to publish. 
S. Y. and N. M. initiated the idea; N. M. designed the study; the JECS study group collected the data; N. M. analysed the data, wrote the paper and had primary responsibility for the final content. K. Kato, M. S., E. S., M. T., A. S., T. K. and K. Kusuhara critically reviewed the draft and provided important intellectual content to the interpretation of the results. All the authors have read and approved the final manuscript.

The authors declare that there are no conflicts of interest.

\section{Supplementary material}

For supplementary material/s referred to in this article, please visit https://doi.org/10.1017/S000711451800291X

\section{References}

1. Alexander BT, Dasinger JH \& Intapad S (2015) Fetal programming and cardiovascular pathology. Compr Physiol 5, 997-1025.

2. Barker DJ (2006) Adult consequences of fetal growth restriction. Clin Obstet Gynecol 49, 270-283.

3. Langley-Evans SC (2001) Fetal programming of cardiovascular function through exposure to maternal undernutrition. Proc Nutr SOC 60, 505-513.

4. Koshy TS, Sara VR, King TL, et al. (1975) The influence of protein restriction imposed at various stages of pregnancy on fetal and placental development. Growth 39, 497-506.

5. Bergmann RL, Bergmann KE \& Dudenhausen JW (2008) Undernutrition and growth restriction in pregnancy. Nestle Nutr Workshop Ser Pediatr Program 61, 103-121.

6. Pinheiro DF, Pinheiro PF, Buratini J Jr, et al. (2013) Maternal protein restriction during pregnancy affects gene expression and immunolocalization of intestinal nutrient transporters in rats. Clin Sci (Lond) 125, 281-289.

7. Langley-Evans SC, Langley-Evans AJ \& Marchand MC (2003) Nutritional programming of blood pressure and renal morphology. Arch Physiol Biochem 111, 8-16.

8. Godfrey K, Robinson S, Barker DJ, et al. (1996) Maternal nutrition in early and late pregnancy in relation to placental and fetal growth. BMJ 312, 410-414.

9. Stevens B, Buettner P, Watt K, et al. (2015) The effect of balanced protein energy supplementation in undernourished pregnant women and child physical growth in low- and middle-income countries: a systematic review and metaanalysis. Matern Child Nutr 11, 415-432.

10. Imdad A \& Bhutta ZA (2012) Maternal nutrition and birth outcomes: effect of balanced protein-energy supplementation. Paediatr Perinat Epidemiol 26, Suppl. 1, 178-190.

11. Merialdi M, Carroli G, Villar J, et al. (2003) Nutritional interventions during pregnancy for the prevention or treatment of impaired fetal growth: an overview of randomized controlled trials. J Nutr 133, 1626s-1631s.

12. Sloan NL, Lederman SA, Leighton J, et al. (2001) The effect of prenatal dietary protein intake on birth weight. Nutr Res $\mathbf{2 1}$, 129-139.

13. Liberato SC, Singh G \& Mulholland K (2013) Effects of protein energy supplementation during pregnancy on fetal growth: a review of the literature focusing on contextual factors. Food Nutr Res 57, 20499.

14. Rasmussen KM \& Yaktine AL (2009) Weight Gain During Pregnancy: Reexamining the Guidelines. Washington, DC: National Academy of Sciences.
15. Watson PE \& McDonald BW (2010) The association of maternal diet and dietary supplement intake in pregnant New Zealand women with infant birthweight. Eur J Clin Nutr 64 , 184-193.

16. Andreasyan K, Ponsonby AL, Dwyer T, et al. (2007) Higher maternal dietary protein intake in late pregnancy is associated with a lower infant ponderal index at birth. Eur J Clin Nutr 61, 498-508.

17. Kawamoto T, Nitta H, Murata K, et al. (2014) Rationale and study design of the Japan environment and children's study (JECS). BMC Public Health 14, 25.

18. Michikawa T, Nitta H, Nakayama S, et al. (2018) Baseline profile of participants in the Japan Environment and Children's Study (JECS). J Epidemiol 28, 99-104.

19. Yokoyama Y, Takachi R, Ishihara J, et al. (2016) Validity of short and long self-administered food frequency questionnaires in ranking dietary intake in middle-aged and elderly Japanese in the Japan Public Health Center-Based Prospective Study for the Next Generation (JPHC-NEXT) protocol area. J Epidemiol 26, 420-432.

20. Sasaki S, Ishihara J \& Tsugane S (2003) Reproducibility of a self-administered food frequency questionnaire used in the 5-year follow-up survey of the JPHC Study Cohort I to assess food and nutrient intake. J Epidemiol 13, S115-S124.

21. Tsugane S, Kobayashi M \& Sasaki S (2003) Validity of the selfadministered food frequency questionnaire used in the 5-year follow-up survey of the JPHC Study Cohort I: comparison with dietary records for main nutrients. J Epidemiol 13, S51-S56.

22. Uehara R, Miura F, Itabashi K, et al. (2011) Distribution of birth weight for gestational age in Japanese infants delivered by cesarean section. J Epidemiol 21, 217-222.

23. Willett WC, Howe GR \& Kushi LH (1997) Adjustment for total energy intake in epidemiologic studies. Am J Clin Nutr $\mathbf{6 5}$, 1220S-1228S; discussion 1229S-1231S.

24. Moore VM, Davies MJ, Willson KJ, et al. (2004) Dietary composition of pregnant women is related to size of the baby at birth. J Nutr 134, 1820-1826.

25. Cuco G, Arija V, Iranzo R, et al. (2006) Association of maternal protein intake before conception and throughout pregnancy with birth weight. Acta Obstet Gynecol Scand 85, 413-421.

26. Switkowski KM, Jacques PF, Must A, et al. (2016) Maternal protein intake during pregnancy and linear growth in the offspring. Am J Clin Nutr 104, 1128-1136.

27. Haste FM, Brooke OG, Anderson HR, et al. (1991) The effect of nutritional intake on outcome of pregnancy in smokers and non-smokers. Br J Nutr 65, 347-354.

28. Lagiou P, Tamimi RM, Mucci LA, et al. (2004) Diet during pregnancy in relation to maternal weight gain and birth size. Eur J Clin Nutr 58, 231-237.

29. Chong MF, Chia AR, Colega M, et al. (2015) Maternal protein intake during pregnancy is not associated with offspring birth weight in a multiethnic Asian population. J Nutr 145 , 1303-1310.

30. Petridou E, Stoikidou M, Diamantopoulou M, et al. (1998) Diet during pregnancy in relation to birthweight in healthy singletons. Child Care Health Dev 24, 229-242.

31. Ota E, Hori H, Mori R, et al. (2015) Antenatal dietary education and supplementation to increase energy and protein intake. Cochrane Database Syst Rev, issue 6, CD000032.

32. Godfrey KM, Sheppard A, Gluckman PD, et al. (2011) Epigenetic gene promoter methylation at birth is associated with child's later adiposity. Diabetes 60, 1528-1534.

33. Ribaroff GA, Wastnedge E, Drake AJ, et al. (2017) Animal models of maternal high fat diet exposure and effects on metabolism in offspring: a meta-regression analysis. Obes Rev 18, 673-686. 
34. Giraudo SQ, Della-Fera MA, Proctor L, et al. (2010) Maternal high fat feeding and gestational dietary restriction: effects on offspring body weight, food intake and hypothalamic gene expression over three generations in mice. Pharmacol Biochem Behav 97, 121-129.

35. Cunha Fda S, Dalle Molle R, Portella AK, et al. (2015) Both food restriction and high-fat diet during gestation induce low birth weight and altered physical activity in adult rat offspring: the "Similarities in the Inequalities" model. PLOS ONE $\mathbf{1 0}$ e0118586.

36. Howie GJ, Sloboda DM, Kamal T, et al. (2009) Maternal nutritional history predicts obesity in adult offspring independent of postnatal diet. J Physiol 587, 905-915.

37. Ogawa K, Jwa SC, Kobayashi M, et al. (2017) Validation of a food frequency questionnaire for Japanese pregnant women with and without nausea and vomiting in early pregnancy. J Epidemiol 27, 201-208.
38. Rifas-Shiman SL, Rich-Edwards JW, Willett WC, et al. (2006) Changes in dietary intake from the first to the second trimester of pregnancy. Paediatr Perinat Epidemiol 20, 35-42.

39. Ogawa K, Morisaki N, Sago H, et al. (2018) Association between women's perceived ideal gestational weight gain during pregnancy and pregnancy outcomes. Sci Rep 8, 11574.

40. Tsukamoto H, Fukuoka H, Inoue K, et al. (2007) Restricting weight gain during pregnancy in Japan: a controversial factor in reducing perinatal complications. Eur J Obstet Gynecol Reprod Biol 133, 53-59.

41. Ikeda N, Takimoto H, Imai S, et al. (2015) Data resource profile: the Japan National Health and Nutrition Survey (NHNS). Int J Epidemiol 44, 1842-1849.

42. Takimoto H, Yoshiike N, Katagiri A, et al. (2003) Nutritional status of pregnant and lactating women in Japan: a comparison with non-pregnant/non-lactating controls in the National Nutrition Survey. J Obstet Gynaecol Res 29, 96-103. 